\title{
Definition, Convention, and Simultaneity: Malament's Result and Its Alleged Refutation by Sarkar and Stachel
}

\author{
Robert Rynasiewicz \\ Department of Philosophy, Johns Hopkins University
}

\begin{abstract}
The question whether distant simultaneity (relativized to an inertial frame) has a factual or a conventional status in special relativity has long been disputed and remains in contention even today. At one point it appeared that Malament (1977) had settled the issue by proving that the only non-trivial equivalence relation definable from (temporally symmetric) causal connectability is the standard simultaneity relation. Recently, however, Sarkar and Stachel (1999) claim to have identified a suspect assumption in the proof by defining a non-standard simultaneity relation from causal connectability. I contend that their critique is based on a misunderstanding of the criteria for the definability of a relation, a misunderstanding that Malement's original treatment helped to foster. There are in fact a variety of notions of definability that can be brought to bear. They all, however, require a condition that suffices to secure Malament's result. The non-standard relation Sarkar and Stachel claim to be definable is not so definable, and, I argue, their proposal to modify the notion of "causal definability" is misguided. Finally, I address the relevance of Malament's result to the thesis of conventionalism.
\end{abstract}

1. Introduction. In his classic paper setting out the special theory of relativity, Einstein (1905) observed that, although the time of an event can be given by the simultaneous reading of a clock in its immediate neighborhood, the times of occurrence of events at different locations cannot be compared without some further arrangement [Festsetzung] (893-4). One has at best a "time" given by the readings of a clock at the one location and a "time" given by those of a clock at the other. A common "time" can be defined by stipulating by definition that the "time" it takes for light to travel from the one to the other is equal to the "time" required for the reverse. From this follows the standard synchronization condition that the two clocks synchronize if, upon sending a light ray from one to the other and reflecting it back to the first, the reading on the clock at the site of reflection exactly splits the difference between the readings on the first clock at departure and at return (894). 
Although the rationale for making some stipulation about the one-way speed of light as a matter of definition was lost on not a few early commentators and textbook writers (see in particular Silberstein (1914, 93-99), Schlick (1920, 1417 ) and Cunningham (1921, 28-31)), Reichenbach (1924, 10) eventually assimilated Einstein's stipulation to the status of a convention in the sense in which Helmholtz and Poincaré had held that geometry is conventional. Grünbaum (1973, 342-69) understood the basis for the Einstein definition to lie in the "fact" that the relation of causal connectability between spacetime events is insufficient for uniquely establishing a strict temporal ordering of events, even from the point of view of a fixed inertial frame of reference.

Malament (1977) directly challenges this alleged "fact." According to Malament, Grünbaum commits himself to the following pair of assertions (293):

(1) The relation of simultaneity relative to an inertial observer is not uniquely definable in terms of the relation $\kappa$ of causal connectability.

(2) Temporal relations are non-conventional if and only if they are so definable.

Malament observes that A. A. Robb (1914) had in effect already shown that the relation of orthogonality on the [affine] space $R^{4}$ with Minkowski inner product is "explicitly, first order definable in terms of $\kappa$ " (296), where the ordered pair $(p, q)$ is in the relation $\kappa$ just in case the inner product of $p-q$ with itself is nonnegative. ${ }^{1}$ Hence, one can define two events $e_{1}, e_{2}$ to be simultaneous relative to inertial observer $O$ if and only if for some (in fact, any) pair $r, s$ of distinct events on the world-line of $O$, the vector $e_{2}-e_{1}$ is orthogonal to $r-s$. This is extensionally equivalent to the standard Einstein synchronization condition above. Malament goes on to show that the only other non-trivial ${ }^{2}$ equivalence relation between events definable from $k$ and $O$ is the universal relation. Hence, assuming that the relation of simultaneity for an inertial observer is a nontrivial equivalence relation smaller than the universal relation, it follows that the standard simulataneity relation is uniquely definable, contra (1) above. By (2), then, it is non-conventional.

Recently, Sarkar and Stachel (1999) have challenged the validity of Malament's result. They claim to have found another (in fact, two other) non-trivial equivalence relations definable for $O$ from $\kappa$. From the fact that these are ruled out by Malament's proof they infer that Malament imposes "an unwarranted physical assumption: that any simultaneity relation must remain invariant under temporal reflections." (208), and argue that this assumption should be relaxed. Spirtes (1981, 171-186) has also claimed that if the restriction of invariance under temporal reflections is lifted, then non-standard simultaneity relations are definable, in fact uncountably many.

The purpose of this note is to explain in what ways Sarkar and Stachel's critique of Malament is misguided. The non-standard simultaneity relation they

\footnotetext{
${ }^{1}$ This assumes the sign convention $(+,-,-,-)$.

${ }^{2}$ In the sense that at least some point on $O$ is equivalent to at least one point not on $O$.
} 
claim to be able to define for $O$ from $\kappa$ is in fact not so definable. Furthermore, Malament's result does not rely on "physical assumptions" either warranted or unwarranted. Unfortunately, the issue is somewhat confused by the fact that Malament gave an incorrect formulation of what it means for a relation to be implicitly definable. Sarkar and Stachel assume that this formulation has the status of a proposal and thus consider it open for criticism whether what they call Malament's "explication" (Sarkar and Stachel, 212) is "physically reasonable" (215). However, just what relations are definable from what is a purely formal, mathematical question and has nothing to do with issues of physical adequacy. There are in fact several, distinct formal notions of definability available from mathematical logic, and it is less than clear specifically which of these Malament had in mind. However, no matter which is adopted, his result remains unaffected, since it relies on a condition strictly weaker than the one he gives, and this weaker condition is a logical consequence of each of these notions of definability. In conclusion, I shall offer a few remarks of the bearing of Malament's result on the issue of the conventionality of simultaneity.

2. Sarkar and Stachel on the Definability of Light Lobes. One of the results due to Robb (1914) is that the relation of lightlike relatedness $\lambda$ between events in Minkowski spacetime is definable from $\kappa$ alone. ${ }^{3}$ Quite clearly then, any relation that is definable using $\lambda$ in addition is definable from $\kappa$ alone. In particular, Sarkar and Stachel claim that the relation " $p$ and $q$ lie on the same half null cone with vertex $e$ " is just so definable in virtue of the following defining condition:

(i) $p$ and $q$ both lie on the light cone with vertex $e$, and

(ii) either $p$ and $q$ are not causally connectable or else there exists a signal connecting them which does not pass through the space-like "elsewhere"

\footnotetext{
${ }^{3}$ The relation $\lambda$ on $R^{4}$ is the set of ordered pairs $\{(p, q) \mid(p-q) \cdot(p-q)=0\}$. The first order formula$$
p \kappa q \wedge \exists r(r \neq p \wedge r \neq q \wedge \forall s(s \kappa p \wedge s \kappa q \rightarrow s \kappa r))
$$

defines $\lambda$ in the structure $\left(R^{4}, \kappa\right)$. For those expert in these matters who might be put off by the lack of complete precision in this section and the next, I offer the following remarks. (For those not expert, the technicalities will explained in section 4.) Above I have used the notion of definability in a structure rather than that of explicit definability in a theory. Malament's locution "explicitly first order definable in terms of $\kappa(300)$ is ambiguous between this and the notion of explicit (first order) definability in a theory. As will be seen later, this formula also explicitly defines $\lambda$ in $T$, where $T$ can be characterized equivalently as either the deductive closure of Robb's axioms or the set of all sentences of the predicate language containing $\kappa$ alone that are true in $\left(R^{4}, \kappa\right)$. Note as well that, following Malament, I have systematically failed to distinguish between ' $\kappa$ ' as a name for the predicate of this language and ' $\kappa$ ' as a symbol for the relation assigned to that predicate as its interpretation in the structure $\left(R^{4}, \kappa\right)$. Also, in order not to disrupt the flow of the discussion, I will, for the time being, continue to use in the body of the text such locutions as "definable from $\kappa$ " despite the ambiguity as to which notion of definability is intended.
} 
of $e$ or through $e$ itself.

The first task is to check whether indeed these conditions can be expressed rigorously using only $\kappa$ and/or $\lambda$, something Sarkar and Stachel do not concern themselves with. The first conjunct (i) is easily rendered by the formula:

(i') $p \lambda e \wedge q \lambda e$.

The second conjunct (ii) requires a bit of finesse. The trouble lies with the invocation of the notion of a causal signal, i.e., a continuous curve whose tangent is never spacelike. Epistemologically (and maybe even ontologically), it certainly makes sense to regard this notion as more primitive than the relation of causal connectability. However, in the game that's at the table, in which causal connectability is the sole primitive, it must, rigorously speaking, be reconstructed from that sole primitive. Actually, for the purposes at hand, it would suffice to find a way of expressing when three events are respectively optically, or inertially collinear, and when so, which of the three is between the other two. Most of this task can be handled in the fashion of Robb via definitions of optical line, parallel optical lines, acceleration plane, inertial line, etc. However, since Robb took the notion of causally after to be his primitive, some additional cunning is needed, particularly in handling relations of betweeness. The details are edifying, but hardly to the point for present purposes.

The second, and main task is to asses what Sarkar and Stachel think follows from this. What bearing does this have on Malament's result? Sarkar and Stachel argue as follows. Temporarily fix the parameter $e$ and let $\Lambda(e)$ be the full light cone centered at $e$.

It follows that there are two such half null cones, with only $e$ in common, that is, $\Lambda_{-}(e) \cup \Lambda_{+}(e)=\Lambda(e)$ and $\Lambda_{-}(e) \cap \Lambda_{+}(e)=\{e\}$. Note that this distinction, once made on the null cone at any point of spacetime, can be consistently carried by parallel transport to the null cone at any other point of spacetime.

Thus $\lambda$ can be used to define two additional simultaneity relations, $\mathrm{Back}_{O, e}$ and $\mathrm{Forw}_{O, e}$, relative to a time-like world line $O$ : two events are simultaneous relative to an event $e$ on $O$ if and only if they lie on $\Lambda_{-}(e)\left[\Lambda_{+}(e)\right]$. Clearly neither relation is "vacuous" in Malament's (297) sense: $e$ is simultaneous to events not on $O$ and not all events are simultaneous. Each is also trivially an equivalence relation. Most importantly, each of the relations can be defined from $\lambda$ alone, since $\Lambda_{-}(e)\left[\Lambda_{+}(e)\right]$ can be so defined. (Note, that since they are definable from $\lambda$, they are also definable from $\kappa$.)

Clearly, something is amiss with Malament's theorem. A correct mathematical result seems to be contradicted by patently good counterexamples. 
What may be patent to them may not be so for everyone. Again, we need to check for rigor. To begin with, there is an obvious slip, though one that is easily correctable. Fix the parameters $O$ and $e$ in the definition of $B a c k_{O, e}$. The resulting equivalence relation has but two equivalence classes, $\Lambda_{-}(e)$ and its complement. Hence, any event not on the past light cone of e is "Back ${ }_{O, e}$ "simultaneous to any other event not on the back light cone of $e$. Clearly this is not what is intended. The proposed simultaneity criterion should yield a oneparameter family of equivalence relations, one for each choice of world line $O$. Having fixed $O$, the events $e$ on $O$ then generate the equivalence classes $[e]$ of the relation defined. So, the term ' $e$ ' should not appear as an additional parameter in the definiens, but at best as a variable quantified over in the definiendum.

The relation Sarkar and Stachel have in mind is in fact given by:

$$
\operatorname{Sim}_{O}^{-}(p, q) \text { iff } \exists e\left(e \in O \wedge p, q \in \Lambda_{-}(e)\right) .
$$

In other words, $p$ and $q$ are back-light-cone simultaneous for world-line $O$ just in case they lie on the back light cone of some event $e$ on $O$ (and similarly for the forward directed counterpart relation $\mathrm{Sim}_{O}^{+}$).

These two relations, however, are not counterexamples; they are not definable from $\lambda$ and/or $\kappa$ alone. The reason is that, although the relation of lying on the same half null cone may be definable, and indeed it can be shown that there exist exactly two half cones, neither one of these cones is itself definable. It is an elementary fallacy to think that just because two things satisfying a certain condition are given, one thereby succeeds in defining either one of them merely by introducing a pair of labels. In the event that this is not completely obvious, it may help to examine some parallel examples.

3. Two Analogues. Consider from group theory Klein's Viergruppe, the four-element group $V$ with the following multiplication table, where $e$ is the identity element. ${ }^{4}$

\begin{tabular}{c|cccc} 
& $\mathrm{e}$ & $\mathrm{a}$ & $\mathrm{b}$ & $\mathrm{c}$ \\
\hline $\mathrm{e}$ & $\mathrm{e}$ & $\mathrm{a}$ & $\mathrm{b}$ & $\mathrm{c}$ \\
$\mathrm{a}$ & $\mathrm{a}$ & $\mathrm{e}$ & $\mathrm{c}$ & $\mathrm{b}$ \\
$\mathrm{b}$ & $\mathrm{b}$ & $\mathrm{c}$ & $\mathrm{e}$ & $\mathrm{a}$ \\
$\mathrm{c}$ & $\mathrm{c}$ & $\mathrm{b}$ & $\mathrm{a}$ & $\mathrm{e}$
\end{tabular}

The automorphisms of $V$ are given by all permutations of $a, b, c$. Although $V$ contains exactly three elements distinct from the identity element, a fact obviously first-order expressible in the language of group theory, none of these

\footnotetext{
${ }^{4}$ Apart from the cyclic group of order four, there is up to isomorphism only one other fourelement group, a fact potentially disguised by some of its realizations. For example, Klein's Viergruppe is also the symmetry group of a line segment in the Euclidean plane, as well as the product group $C_{2} \times C_{2}$, where $C_{n}$ is the cyclic group of order $n$.
} 
elements is individually definable. (The language can be taken to include, besides the usual logical symbols, an individual constant symbol for the identity element and a function symbol for group multiplication.) To drive home the general point, we can construct a tighter analogy with the light cone case (with fixed vertex $e$ ) as follows. The entire group $V$ will play the role of $\Lambda(e)$. Next define a relation $x R y$ (which you are invited to read as ' $x$ lies on the same "lobe" as $y^{\prime}$ ) by

$$
x R y \text { iff }((x \circ y=x) \vee(x \circ y=y)) .
$$

The relation $R$ generates the three "lobes" $\{e, a\},\{e, b\},\{e, c\}$, which as a triplet you are invited to think of as the analogue of the pair of cones with vertex $e$. Note, again, that there exist exactly three "lobes" sharing the identity element is first-order expressible. ${ }^{5}$ Now, in order for the lobe, say $\{e, a\}$ to be definable, there must exist a group-theoretic equation in one free variable which is solved by $e$ and $a$ alone. However, from the fact that the automorphisms of $V$ are the permutations of $a, b, c$, it follows that if $a$ is a solution to an equation, then so are $b$ and $c$. Hence, no individual lobe is definable. I hope this helps to make it more compelling why it is that simply introducing labels gets one no closer to definitions.

A second example, which matches the light lobes case in cardinality, relies only on the topology of the real line. Let betweeness be given as the only primitive relation on $R^{1}$ and pick some distinguished point of $R^{1}$, for the sake of definiteness the base $e$ of the natural logarithm, as the only other primitive. Now define the relation $S^{e} x y$ by

$$
S^{e} x y \text { iff } \neg e B x y,
$$

where $z B x y$ is to be read " $z$ lies between $x$ and $y . "$ Hence $S^{e} x y$ just in case $x$ and $y$ lie on the same ray with end point $e$. Think of this binary relation as the analogue of " $p$ and $q$ lie on the same half null cone with vertex $e$ ". Obviously, there are exactly two rays of the real line with end point $e$ a fact expressible even without recourse to a many sorted language or higher order quantification. ${ }^{6}$ Think of these two rays as counterparts of the two lobes of the light cone with vertex at event $e$. Of course, we might introduce labels, say $R A Y_{-}$and $R A Y_{+}$ "merely to distinguish them" (Sarkar and Stachel, 213), without claiming to know which denotes which ray or in anyway introducing an orientation on the real line. But again, this in no way establishes that each individual ray is

\footnotetext{
${ }^{5}$ Transcribe into first order notion the following: there exist at least three elements, none on the same lobe as another, such that any other element is on the same lobe as one of the three.

${ }^{6}$ Consider the first-order sentence

$\exists x \exists y\left(x \neq y \wedge x \neq e \wedge y \neq e \wedge S^{e} x e \wedge S^{e} y e \wedge \neg S^{e} x y \wedge \forall z\left(z \neq x \wedge z \neq y \wedge z \neq e \rightarrow S^{e} z x \vee S^{e} z y\right)\right)$.
}

A first order sentence of the same form then expresses the fact that there are exactly two half light cones at $e$ by substituting the analogous predicate and relativizing the quantifiers to the set of events lightlike related to $e$. 
definable from the betweeness relation (and $e$ ) alone. What is needed for each is a predicate expression (involving only $e$ and $B$ ) in one free variable satisfiable by all and only points on the ray to be defined. As will shortly become evident, it is provable by symmetry arguments that no such expressions exist.

In presenting these examples, I hope to have tapped intuitions about the conditions under which a given relation or object is definable. But I do not want the case to rest solely with that. There is a standard literature on definability that backs those intuitions. Before turning to this, it may be instructive to consider just what does transpire in the familiar form of reasoning which proceeds: "We have shown there are exactly two things which are such-and-such. Call them $c_{1}$ and $c_{2}$ respectively. It now follows that ...."

Let $\varphi(x)$ be a predicate expression in one free variable $x$, and suppose it has been established that there are exactly two things satisfying $\varphi(x)$. What is the effect of introducing arbitrary tags $c_{1}, c_{2}$ in subsequent reasoning? The upshot of the move is to make available the following assumptions: $\varphi\left(c_{1}\right), \varphi\left(c_{2}\right), c_{1} \neq c_{2}$, and finally $\forall x\left(\varphi(x) \rightarrow\left(x=c_{1} \vee x=c_{2}\right)\right)$. If one then succeeds in deriving a sentence $\sigma$ not containing either $c_{1}$ or $c_{2}$, then one knows that $\sigma$ can be proven, in a probably much more obtuse fashion, without the introduction of $c_{1}$ and $c_{2}$ and their associated assumptions. Those of you stuck with teaching logic term after term will recognize this as nothing other than a generalized instance of the rule of inference called Existential Instantiation, according to which, having shown $\exists x \varphi(x)$, one says "let us introduce a name $c$ for an arbitrarily selected individual satisfying $\varphi(x)$ " and proceeds to employ the assumption $\varphi(c)$ in subsequent reasoning. No one, of course, thinks that one has thereby succeeded in defining one of the entities that happens to satisfy $\varphi(x)$.

I turn now to the standard literature on definability.

4. Notions of Definability. So far I have been somewhat sloppy with regard to certain techinical distinctions, among them, just what notion of definability is in play. The preceding discussion, however, is most naturally construed as addressing the notion of definability in a structure.

Let $\boldsymbol{A}$ be a structure for a first-order language $\mathcal{L}, R$ an $n$-ary relation on the domain of discourse of $\boldsymbol{A}$, and $s$ an assignment of elements of the domain of discourse of $\mathfrak{A}$ to the individual variables of $\mathcal{L}$. Then $R$ is said to be definable in $\mathfrak{A}$ just in case the following: there is a formula $\varphi$ of $\mathcal{L}$ whose free individual variables are among $x_{1}, \ldots, x_{n}$ such that for any $s, \mathfrak{A}$ satisfies $\varphi$ with $s$ if and only if the $n$-tuple $\left\langle s\left(x_{1}\right), \ldots, s\left(x_{n}\right)\right\rangle$ is a member of $R$ (Enderton 1972, 88).

In order to illustrate how this applies in the above examples, we need to be slightly more precise in our notation. For the group-theoretic example, the non-logical vocabulary contains the two-place function symbol $\circ$ for group composition and an individual constant symbol $e$ for the group identity. ${ }^{7}$ The Vier-

\footnotetext{
${ }^{7}$ Although the group identity is definable in any model of group theory, I include $e$ as a primitive of the language for expository purposes.
} 
gruppe $\mathfrak{V}$, as a structure for this language, consists of the set $V=\left\{e^{\mathfrak{V}}, a, b, c\right\}$ together with the binary operation $0^{\mathfrak{V}}$ specified by the multiplication table. (The horrendous notation is necessary to distinguish the identity symbol from the identity element and the function symbol from the symbol's interpretation.) Since, technically speaking, the relation $R$ is not a predicate symbol, but a subset of $V \times V$, the definition of $R$ in this example should read:

$$
<a_{1}, a_{2}>\in R \text { iff } \mathfrak{V} \models((x \circ y=x) \vee(x \circ y=y)) \quad\left[a_{1}, a_{2}\right],
$$

where $\mathfrak{V} \models \varphi(x, y)\left[a_{1}, a_{2}\right]$ means that $\mathfrak{V}$ satisfies $\varphi(x, y)$ with $s$ for any assingment $s$ of elements of $V$ to variables such that $s(x)=a_{1}$ and $s(y)=a_{2}$.

The question as to whether a given subset (e.g., a "lobe") of the Viergruppe is first order definable in the group is now just the question as to whether there is a defining formula with one free variable. The following lemma can be used to establish that certain subsets (or relations in general) are not definable in a given structure.

First Automorphism Lemma. The (in general $n$-ary) relation $R$ is definable in $\mathfrak{A}$ only if $R$ is preserved under all automorphisms of $\mathfrak{A}$.

It immediately follows that none of the three "lobes" of the Viergruppe is definable in the group since they are not invariant under group automorphisms. Similarly with the two rays from the second example. Reflection through the point $e$ preserves betweeness on the real line, and hence is an automorphism of that structure. But neither ray is preserved under reflections.

One might ask about higher-order definability in these examples. Fortunately, it is not necessary to go into the details of the conditions for second or higher order logic. The above automorphism lemma holds under generalization to higher orders.

There are two other standard notions of definability, both variants of the idea of definability in a given theory, where a theory is understood to be a deductively closed set of sentences. Here, the distinction between a predicate symbol and its interpretation as a relation in a given model of the theory is crucial since what is involved is really the definition of the intension of a predicate (or function) symbol. By the intension of an $n$-ary predicate in a theory, we mean a function that assigns to each model of the theory an $n$-ary relation on the domain of discourse of the model. When it is said that a given relation is definable in a theory, what is meant is that the intension of an associated predicate symbol is definable. It is also taken for granted that the associated predicate is part of the vocabulary in which the theory is formulated. However, so much nit-pickiness eventually becomes cumbersome and, despite the potential for type confusions, it is simply said that: an $n$-ary relation $P$ is explicitly definable in theory $T$ if and only if in the language of $T$ there exists a formula $\theta\left(x_{1}, \ldots, x_{n}\right)$, with variables $x_{1}, \ldots, x_{n}$ free but in which $P$ does not occur, such that the sentence

$$
\forall x_{1} \ldots \forall x_{n}\left(P x_{1} \cdots x_{n} \leftrightarrow \theta\left(x_{1}, \ldots, x_{n}\right)\right)
$$


is a theorem of $T$. In order to relate this to the previous notion of definability in a structure, we adopt the following notational convention. If $\mathfrak{A}$ is a structure for the language of $T$ minus the $n$-ary predicate $P$ and $R$ is an $n$-ary relation on the domain of discourse of $\boldsymbol{A}$, then $(\boldsymbol{A}, R)$ is a structure for the full language of $T$ in which $R$ is the extension of $P$. The following is not difficult to prove: if $P$ is explicitly definable in $T$, then the relation $R$ is definable in $\mathfrak{A}$ for any model $(\mathfrak{A}, R)$ of $T$. In fact, the open formula $\theta\left(x_{1}, \ldots, x_{n}\right)$ that explicitly defines $P$ in $T$ defines $R$ in $\mathfrak{A}$. An immediate consequence is a corollary to the above automorphism lemma.

Second Automorphism Lemma. $P$ is explicitly definable in theory $T$ only if for each model $(\mathfrak{A}, R)$ of $T$ the extension $R$ of $P$ is preserved under automorphisms of $\boldsymbol{A}$.

The other standard notion of definability in a theory is that of implicit definability. ${ }^{8}$ Using the above notation, $P$ is said to be implicitly definable in $T$ just in case if $(\mathfrak{A}, R)$ and $\left(\mathfrak{A}, R^{\prime}\right)$ are any two models of $T$, then $R=R^{\prime}$. A result due to Beth is that for first order logic $P$ is implicitly definable in $T$ if and only if $P$ is explicitly definable in $T$. For higher order logics only the "if" direction of the biconditional holds generally. Nonetheless, a direct and general connection between implicit definability and model automorphism is available, so general in fact that it applies directly to classes of structures without the intermediary of deductively closed sets of sentences if we replace the models of $T$ with elements of a class $K$ of structures in the characterization of implicit definability.

Third Automorphism Lemma. If $P$ is implicitly definable in $K$ and $K$ is closed under isomorphism, then the extension $R$ of $P$ in any member $(\mathfrak{A}, R)$ of $K$ is preserved under automorphisms of $\mathfrak{A}$.

The proof is rather immediate. Let $(\mathfrak{A}, R)$ be a member of $K$ and $\phi$ an automorphism of $\mathfrak{A}$. Letting $\phi$ act on $(\mathfrak{A}, R)$ yields the isomorphic structure $(\phi * \mathfrak{A}, \phi * R)$, which is also a member of $K$ if $K$ is closed under isomorphism. However, since $\phi$ is an automorphism of $\mathfrak{A},(\phi * \mathfrak{A}, \phi * R)=(\mathfrak{A}, \phi * R)$. Thus, if $P$ is implicitly definable in $K,(\mathfrak{A}, \phi * R)=(\mathfrak{A}, R)$.

5. Definable Relations in Minkowski Spacetime. The punch line is now this. No matter what notion of definability is in question, the preservation of a relation under automorphisms of the structure or structures in question is a necessary condition for the definability of the relation. In the case of Minkowski spacetime, these automorphisms include temporal reflections and thus render futile any attempt to define an individual half cone in the absence of a temporal

\footnotetext{
${ }^{8}$ The notion of implicit definability dealt with here should not be confused with that in the writings of Schlick and Reichenbach on the foundations of mathematics inspired by Hilbert's axiomatization of geometry.
} 
orientation. A fortiori the proposed simultaneity relation $\mathrm{Sim}_{O}^{-}$(or $\mathrm{Sim}_{O}^{+}$) is not definable "in terms of $\kappa$ (and $O$ ) alone" whether this is parsed as meaning definable in the structure $\left(R^{4}, \kappa, O\right)$, or as implicitly definable in the class of structures isomorphic to $\left(R^{4}, \kappa, O, \operatorname{Sim}_{O}^{-}\right)$, or as explicitly definable in the set of first or any higher order sentences true in $\left(R^{4}, \kappa, O, \operatorname{Sim}_{O}^{-}\right)$.

So, it might be asked, why not simply provide Minkowski spacetime with a temporal orientation at the outset? After all, Robb took a (temporally) asymmetric causal connectability relation as his primitive, and $\mathrm{CP}$ violation in weak interactions certainly suggests that fundamental laws will not be time-reversal invariant. This is what Sarkar and Stachel do in effect by proposing an alternative "definition" of definability. It is unclear, though, what they think is the upshot of this move.

Part of the difficulty stems from a bit of terminology that slips in innocently towards the beginning of their paper, but is quickly perverted into an expression that is either pleonastic or pernicious. In order to talk about those mappings of $R^{4}$ to itself that preserve the relation of causal connectability, Malament introduces the term of art "causal automorphism" (297). Sarkar and Stachel describe Malament as "explicat[ing] the notion of "causally definable" " (212). As far as I have been able to determine, the latter expression never occurs in Malament's paper. Nonetheless, it could serve as reasonable shorthand for "definable from $\kappa$." However, within the span of a paragraph Sarkar and Stachel introduce the further locution "causally definable from $\kappa$ and $O$," which appears time and again in what follows without further elucidation. As a substitute for "causal automophism" in the sense just explained, this is pleonistic - "causally definable from $O$ " suffices. If some other reading is intended, it is pernicious for then there is no precedent whatsoever for what it might mean.

No doubt Malament can be accused of having invited confusion by misstating the condition for definability in Minkowski spacetime. He writes that a relation is "implicitly definable from $\kappa$ " IF and only if it is preserved under all causal automorphisms (297). ${ }^{9}$ This led Spirtes (1981), on the assumption that preservation under causal automorphisms is sufficient for definability, to the conclusion that in a temporally oriented Minkowski spacetime there are uncountably many non-standard simultaneity relations definable from $\kappa$ and the temporal orientation. ${ }^{10}$ Malament's proof, it should be stressed, requires only the "only if" direction. Inclusion of the "if" direction yields a criterion far too promiscuous to of general service. That is, no further interesting sense of "definability" is to be had with the criterion: $R$ is definable in structure $\mathfrak{A}$ iff $R$ is invariant under all automorphisms of $\mathfrak{A}$. To see this, consider the standard model of arithmetic. It has no non-trivial automorphisms. Hence, it follows from the criterion that any arithmetic relation is definable, a consequence that certainly begs the question in what sense one would at all want to

\footnotetext{
${ }^{9}$ Emphasis mine.

${ }^{10} \mathrm{As}$ far as I am aware, the backward and forward light cone relations are the only two additional equivalence relations definable in a temporally oriented Minkowski spacetime.
} 
speak of "definability."

To sum up, Sarkar and Stachel's proposal to redefine the notion "relation $R$ is causally definable from $\kappa$ and $O$ " is doubly misguided. First, we have no idea what qualification the term "causally" is supposed to add. Second, as an instance of the suggestion to formulate some alternative notion of definability, it falls prey to the sorts of worries just raised in connection with arithmetic.

6. Conclusion. The validity of Malament's result remains unaffected by the considerations and objections raised by Stachel and Sarkar. Despite what they say, the only assumption Malament requires is that the relation in question be a non-trivial equivalence relation strictly smaller than the universal relation.

As to the relevance of Malament's result for the issue of the conventionality of distant simultaneity in special relativity, there remain a number of questions. One is whether simultaneity must be an equivalence relation. Both Redhead $(1993,114)$ and Grünbaum (private communication) have suggested it need not be assumed to be. But this begs two confusions. First, if the support for this move comes from the fact that "topological" simultaneity is not transitive, then it rests on conflating simultaneity (full stop) with simultaneity relative to an inertial observer. That the latter should be an equivalence relation is no more than the requirement that the inertial observer have a criterion (conventional or otherwise) for assigning a time coordinate to each event of spacetime. Second, that simultaneity relative to an inertial observer is an equivalence relation does not entail that the criterion used is independent of the "position" of the inertial observer and hence generalizes immediately to a criterion for simultaneity with respect to the inertial frame of all co-moving observers. If by chance it does, then the criterion of synchronization can be said to be (symmetric and) transitive among all clocks stationed at various "points" in the inertial frame. ${ }^{11}$ But Malament's result does not presuppose this stronger requirement.

Another question is whether the conventionalist is committed to assertion (2) above, i.e., that temporal relations are non-conventional if and only if they are uniquely definable from causal connectibility. Here it should be noted that in general there are perhaps a half dozen distinct forms of conventionalism (Rynasiewicz, 1999). So, whether or not the conventionalist is committed to (2) does not admit a short answer. ${ }^{12}$

The most serious question, however, is this. Described as neutrally as possible, what Malament establishes is that the only (interesting) equivalence relation definable from $\kappa$ and $O$ is that of lying on the same hypersurface spacetimeorthogonal to $O$. Now, as silly or contentious as it may sound, we should ask,

\footnotetext{
${ }^{11}$ See Reichenbach 1958, 168: "One should not confuse ... 'transitivity of simultaneity' with 'transitivity of simultaneity according to the same rule of synchronization'." Emphasis in the original.

${ }^{12}$ In private communication, Grünbaum denies that he commits himself to (2).
} 
what does spacetime orthogonality have to do physically with simultaneity? The force of the question is more easily recognized if reframed as follows. Suppose an inertial observer emits a light pulse in all directions. Consider the intersection of the resulting light cone with some subsequent hypersurface orthogonal to the observer. Does causal connectibility (plus $O$ if you like) completely determine the spatial geometry of the light pulse on that hypersurface in the absence of some stipulation as to the one-way velocity of light? If not (and I urge you to think not), then relative simultaneity does involve a conventional component corresponding to a degree of freedom in choosing a $(3+1)$-dimensional representation of an intrinsically four-dimensional geometry.

Acknowledgments. I would like to thank John Earman, Domenico Giulini, and Adolf Grünbaum for comments on earlier drafts of this essay.

\section{REFERENCES}

Chang, C. C. and H. J. Keisler (1973), Model Theory. Amsterdam: North Holland.

Cunningham, E. (1921), Relativity, the Electron Theory, and Gravitation, 2nd edition. London: Longmans, Green and Co.

Einstein, Albert (1905), "Zur Elektrodynamik bewegter Körper", Annalen der Physik 17: 891-921.

Enderton, Herbert B. (1972), A Mathematical Introduction to Logic. San Diego: Academic Press

Grünbaum, Adolf, (1973), Philosophical Problems of Space and Time, 2nd edition. Dordrecht: Reidel.

Malament, David (1977), "Causal Theories of Time and the Conventionality of Simultaneity", Noûs 11: 293-300.

Redhead, Michael (1993), "The Conventionality of Simultaneity". In Philosophical Problems of the Internal and External Worlds. Edited by John Earman, Allen I. Janis, Gerald Massey, and Nicholas Rescher. Pittsburgh: University of Pittsburgh Press.

Reichenbach, Hans (1958), The Philosophy of Space 8 Time, New York: Dover.

Robb, A. A. (1914), A Theory of Time and Space. Cambridge: Cambridge University Press.

Rynasiewicz, Robert (1999). "Varieties of Conventionality," Volume of Abstracts, International Union of History and Philosophy of Science, and 11th International Congress of Logic, Methodology and Philosophy of Science. Edited by Jacek Cachro and Katarzyna Kijania-Placek. Cracow: Kopiorama. Page 329.

Sarkar, Sahotra and John Stachel (1999), "Did Malament Prove the Non-Conventionality of Simultaneity in the Special Theory of Relativity?", Philosophy of Science 66: $208-220$. 
Schlick, Moritz (1920), Raum und Zeit in der gegenwärtigen Physik, 3rd edition. Berlin: Julius Springer.

Silberstein, L. (1914), The Theory of Relativity. London: Macmillan.

Spirtes, Peter L. (1981), Conventionalism and the Philosophy of Henri Poincaré. Ph. D. Dissertation, Department of the History and Philosophy of Science, University of Pittsburgh. 